\title{
E3 SUMO-Protein Ligase ZNF451
}

National Cancer Institute

\section{Source}

National Cancer Institute. E3 SUMO-Protein Ligase ZNF451. NCI Thesaurus. Code C142208.

E3 SUMO-protein ligase ZNF451 (1061 aa, 121 kDa) is encoded by the human ZNF451 gene. This protein is involved in the sumoylation of protein substrates. 